\title{
A new perspective on appetite control: protein tyrosine phosphatase Ib (ptplb) inactivation by oxidative phytochemicals
}

\section{Obesity and leptin}

Obesity and overweight are resulted from poor control on appetite. A major part of appetite control relies on leptin and its receptor signal pathway (Figure 1). One obvious evidence supporting this idea can be observed from the mutation on leptin ( $o b$ gene in obese mice) or leptin receptor ( $d b$ gene in diabetic mice). C57B1 mice with $o b / o b$ gene will develop obesity while mice with $d b / d b$ gene will develop obesity and diabetes. The recessive $o b$ gene mutation is due to an early termination on the leptin gene transcript. ${ }^{1}$ A replacement therapy on $o b / o b$ mice with full length leptin resulted in reduced appetite and reduction in body weight. ${ }^{2}$

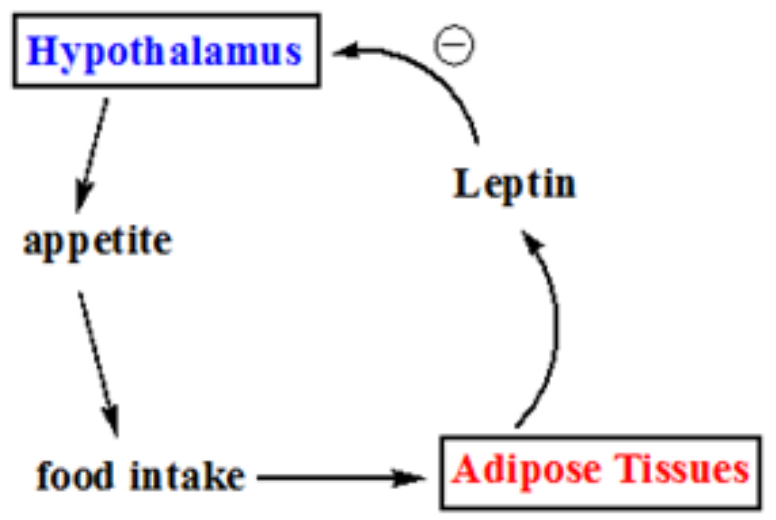

Figure I Leptin feedback on appetite control. Leptin is secreted by adipose tissues and acting as a feedback hormone to suppress appetite signal originated from the hypothalamus.

\section{Leptin signal pathway and PTP I B}

The leptin signal pathway relies on Janus kinase (JAK) and signal transducer/activator of transcription (STAT) proteins to propagate its signal. When leptin binds to its receptor, JAK2 is activated to phosphorylate leptin receptor and STAT3. The phosphorylated STAT3 dimerize and become capable of modulating gene expression in the nucleus. The JAK/STAT signal is modulated by a negative regulator, protein tyrosine phosphatase $1 \mathrm{~b}$ (PTP1B), which dephosphorylates STAT3 and thus renders STAT3 back to its ground state. ${ }^{3,4}$ Incidentally, insulin signal is also modulated by PTP1B. Insulin receptor activation results in phosphorylation on insulin receptor itself and its substrate, insulin receptor substrate (IRS). Both insulin receptor and IRS are modulated by PTP1B. Therefore, a potent PTP1B inhibitor has been proposed as a dual function inhibitor for treating obesity (leptin pathway) and diabetes (insulin pathway), as illustrated in Figure 2.

\section{Insulin signal pathway and $\mathrm{H}_{2} \mathrm{O}_{2}$ formation}

Activated insulin receptor can provoke several different pathways. One interesting but still poorly understood pathway is the activation
Volume 7 Issue 3 - 2017

\section{Henry J Tsai,' Ching Chu Chen ${ }^{2}$}

'Department of Health and Nutrition, Asia University, Taiwan

${ }^{2}$ Internal Medicine, China Medical University, Taiwan

Correspondence: Henry J Tsai, Department of Health and Nutrition, Asia University, Taiwan, Email henrytsai@asia.edu.tw

Received: August 28, 2017 | Published: September 12, 2017

of NADPH oxidase (NOX) which results in an increase in oxygen superoxide. ${ }^{5,6}$ Oxygen superoxide is then converted to hydrogen peroxide $\left(\mathrm{H}_{2} \mathrm{O}_{2}\right)$ by superoxide dismutase (SOD). It has been shown that endogenous $\mathrm{H}_{2} \mathrm{O}_{2}$, arisen from insulin stimulation, can inactivate PTP1B, which in turns potentiates insulin signal transiently, ${ }^{7,8}$ as illustrated in Figure 2.

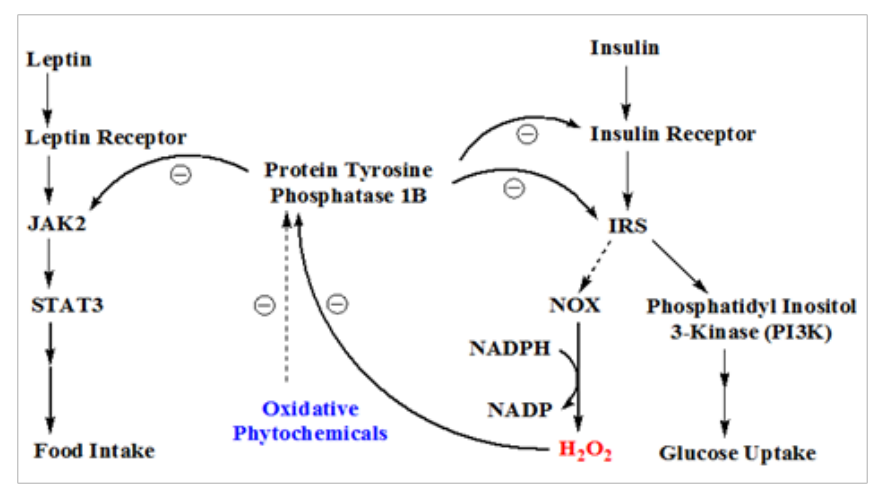

Figure 2 The role of protein tyrosine phosphatase $\mathrm{Ib}$ in leptin and insulin signal pathways. Leptin signal is passed on by JAK2 and STAT3, while insulin signal is mediated by insulin receptor substrate (IRS). IRS can in turn activate phosphatidyl inositol 3-kinase (PI3K) leading to enhanced glucose uptake and/ or upregulated NADPH oxidase (NOX) which leads to an increase of hydrogen peroxide formation. PTPIB is a negative regulator which dephosphorylates JAK2 in leptin signal pathway or insulin receptor/IRS in insulin signal pathway. The endogenous $\mathrm{H}_{2} \mathrm{O}_{2}$ can inactivate protein tyrosine phosphatase $\mathrm{Ib}$ (PTPIB) transiently. Some oxidative phytochemicals can augment the oxidative potential of $\mathrm{H}_{2} \mathrm{O}_{2}$.

\section{Reversible inactivation of PTP I B by $\mathrm{H}_{2} \mathrm{O}_{2}$}

PTP1B is a phosphoryl hydrolase catalyzed by a cysteine residue. During the catalysis, the catalytic cysteine is deprotonated to thiolate anion which becomes a nucleophile. The cysteine thiolate anion is susceptible to $\mathrm{H}_{2} \mathrm{O}_{2}$ oxidation to sulphenic acid (-SOH) which renders PTP1B inactive..$^{9,10}$ The sulphenic group on cysteine will condense with its adjacent amine group of $n+1$ peptide residue to become a sulphenylamide, resulting in an isothiazolidine ring on the peptide 
backbone..$^{11}$ At the sulphenylamide stage, PTP1B enzyme activity can be restored by thiol reducing agents, e.g., glutathione or dithiothreitol, to its active thiolate anion stage. Thus, a mild oxidation on the catalytic cysteine of PTP1B is considered reversible, although the oxidation itself involves formation of covalent bonds. If the sulphenic group on PTP1B is further oxidized to sulphinic acid $\left(-\mathrm{SO}_{2} \mathrm{H}\right)$ or sulphonic acid $\left(-\mathrm{SO}_{3} \mathrm{H}\right)$, PTP1B enzyme activity is lost irreversibly, as illustrated in Figure 3.

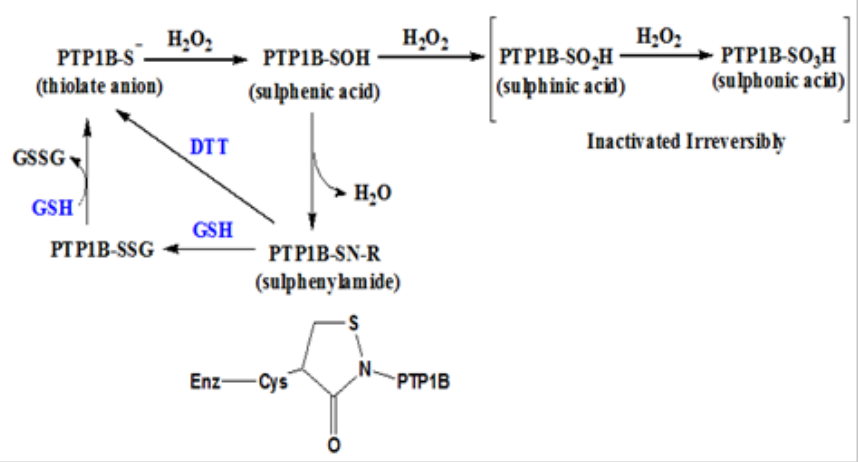

Figure 3 Reversible and irreversible oxidation of cysteine thiol on PTPIB. The thiol or thiolate anion of catalytic cysteine on PTPIB can be oxidized to a sulphenic acid form which can condense with an amine group to form a sulphenylamide resulting in an isothiazolidine ring on the peptide backbone. The sulphenylamide can be reduced by thiol reducing agents like dithiothreitol (DTT) or glutathione (GSH). If the sulphenic acid is further oxidized to sulphinic acid or sulphonic acid, they cannot be reduced back to the thiol form in vivo.

\section{Difficulty on PTP I B inhibitors}

Since the PTP1B gene knocked out mice was published in 1999, ${ }^{12}$ many scientists have been seeking a potent PTP1B inhibitor, which in theory can treat diabetes, overweight or even certain form of her2 ${ }^{+}$ breast cancer (her2 oncogene is also regulated by PTP1B). ${ }^{13}$ The difficult part of finding a satisfactory PTP1B inhibitor is because T cell PTP (TCPTP) isozyme bears a very similar active site contour. So PTP1B inhibitors are not able to distinguish these two PTP isozymes apart. ${ }^{14,15}$ As TCPTP is important for maintaining immune system function, mice lack TCPTP will suffer immune deficiency or even lethality. ${ }^{16}$ It is paramount to inhibit PTP1B but not TCPTP. For example, a recent PTP1B inhibitor ${ }^{17}$ with a selectivity of 30 -fold over TCPTP is not good enough for pharmaceutical purpose, because pharmaceutical reagents require an isozyme selectivity of 1000 fold or greater ( $>3$-order difference in $\mathrm{IC}_{50}$ ) to be considered safe.

\section{Oxidative phytochemicals to augment $\mathrm{H}_{2} \mathrm{O}_{2}$ inactivation on PTP |B}

Since PTP1B can be inactivated transiently by endogenous $\mathrm{H}_{2} \mathrm{O}_{2}$, while PTP1B specific inhibitors are not ready for medical use, an alternative approach of inhibiting PTP1B may be using phytochemicals to mimic the action of $\mathrm{H}_{2} \mathrm{O}_{2}$. What compounds possess such oxidative power as $\mathrm{H}_{2} \mathrm{O}_{2}$ ? Isothiocyanate compounds found in cruciales are capable of inactivating PTP1B. ${ }^{18}$ More recently, guava leaf extract from our laboratory suggest that polar phytochemicals in the guava leaf extract are also capable of inactivating PTP1B enzyme with stronger reactivity (20-80 folds higher pseudo-first reaction rates than those of isothiocyanates). While the identities of these compounds have not been identified, they do show a hypoglycemic efficacy when fed to healthy mice as well as a supplementary effect to insulin stimulated glucose uptake in adipocytes (presumably through an inactivation on PTP1B but elucidated on the insulin pathway). These results would suggest guava leaf extract, i.e., guava leaf tea, may have an appetite suppressing effect because of its oxidative potential on PTP1B.

\section{Acknowledgements}

None.

\section{Conflicts of interest}

The author declares no conflict of interest.

\section{References}

1. Zhang Y, Proenca R, Maffei M, et al. Positional cloning of the mouse obese gene and its human homologue. Nature. 1994;372(6505):425-432.

2. Paz Filho G, Wong ML, Licinio J. Ten years of leptin replacement therapy. Obes Rev. 2011;12(5):315e-323e.

3. Cheng A, Uetani N, Simoncic PD, et al. Attenuation of leptin action and regulation of obesity by protein tyrosine phosphatase 1B. Dev Cell. 2002;2(4):497-503.

4. Pandit R, Beerens S, Adan RAH. Role of leptin in energy expenditure: the hypothalamic perspective. Am J Physiol Regul Integr Comp Physiol. 2017;312(6):938R-947R.

5. Goldstein BJ, Mahadev K, Wu X, et al. Role of insulin induced reactive oxygen species in the insulin signaling pathway. Antioxid Redox Signal. 2005;7(7-8):1021-1031.

6. Ahmadinejad F, Geir Møller S, Hashemzadeh Chaleshtori M, et al Molecular Mechanisms behind Free Radical Scavengers Function against Oxidative Stress. Antioxidants (Basel). 2017;6(3):51-55.

7. Mahadev K, Zilbering A, Zhu L, et al. Insulin-stimulated hydrogen peroxide reversibly inhibits protein-tyrosine phosphatase $1 \mathrm{~b}$ in vivo and enhances the early insulin action cascade. $J$ Biol Chem. 2001;276(24):21938-21942.

8. Mistry RK, Brewer AC. Redox Dependent Regulation of Sulfur Metabolism in Biomolecules: Implications for Cardiovascular Health. Antioxid Redox Signal. 2017;2(1):1-4.

9. Salmeen A, Andersen JN, Myers MP, et al. Redox regulation of protein tyrosine phosphatase $1 \mathrm{~B}$ involves a sulphenyl amide intermediate. Nature. 2003;423(6941):769-773.

10. Dagnell M, Pace PE, Cheng Q, et al. Thioredoxin reductase 1 and NADPH directly protect protein tyrosine phosphatase $1 \mathrm{~B}$ from inactivation during H2O2 exposure. J Biol Chem. 2017;292(35):14371-14380.

11. Van Montfort RL, Congreve M, Tisi D, et al. Oxidation state of the active-site cysteine in protein tyrosine phosphatase 1B. Nature. 2003;423(6941):773-777.

12. Elchebly M, Payette P, Michaliszyn E, et al. Increased insulin sensitivity and obesity resistance in mice lacking the protein tyrosine phosphatase1B gene. Science. 1999;283(5407):1544-1548.

13. Julien SG, Dubé N, Read M, et al. Protein tyrosine phosphatase 1B deficiency or inhibition delays ErbB2-induced mammary tumorigenesis and protects from lung metastasis. Nat Genet. 2007;39(3):338-346.

14. Iversen LF, Moller KB, Pedersen AK, et al. Structure determination of T cell protein-tyrosine phosphatase. J Biol Chem. 2002;277(22):1998219990.

15. Tiganis T. PTP1B and TCPTP-nonredundant phosphatases in insulin signaling and glucose homeostasis. FEBS J. 2013;280(2):445-458.

16. Ibarra Sánchez MJ, Simoncic PD, Nestel FR, et al. The T-cell protein tyrosine phosphatase. Semin Immunol. 2000;12(4):379-386. 
17. Zhang R, Yu R, Xu Q, et al. Discovery and evaluation of the hybrid of bromophenol and saccharide as potent and selective protein tyrosine phosphatase 1B inhibitors. Eur J Med Chem. 2017;134:24-33.
18. Lewis $\mathrm{SM}, \mathrm{Li} \mathrm{Y}$, Catalano MJ, et al. Inactivation of protein tyrosine phosphatases by dietary isothiocyanates. Bioorg Med Chem Lett. 2015;25(20):4549-452. 